\title{
Advances in detecting and responding to threats from bioterrorism and emerging infectious disease
}

\author{
Lisa D Rotz \& James M Hughes
}

Much progress has been made in recent years to strengthen local, state, national and international capacities to detect and respond to bioterrorism events and naturally occurring outbreaks of disease. New tools and systems are available to estimate the potential impact of a biological event and predict resource needs for effective response, enable earlier detection of an attack or outbreak, enhance diagnostic capacity and facilitate rapid intervention to mitigate the impact of an event on a community. These advances have required new approaches to preparedness, planning and surveillance, as well as new partnerships and collaborations across a range of disciplines. We examine some of these developments, discuss potential uses and limitations of these approaches, and identify priorities for the future.

\begin{abstract}
Addressing the problem of intentionally disseminated infectious agents is a complex endeavor, requiring new approaches to public health surveillance, as well as new partnerships and collaborations across a broad range of specialties. These collaborations extend beyond the clinical and research communities to include experts in areas such as animal health, agriculture, environmental science, information technology, emergency response and law enforcement. Further complicating this rapidly evolving field is the lack of an evidence base to support recommended actions. Despite these limitations, much progress has been made in recent years to improve local, state, national and international efforts to recognize and respond to a bioterrorism threat and to detect naturally occurring disease outbreaks, ensuring the use of newly developed and existing resources. Here, we examine some of these developments, discuss potential uses and limitations of new and established systems and other capabilities, and help identify future needs.
\end{abstract}

\section{Preparedness}

Planning is an essential part of bioterrorism preparedness, necessary for outlining actions, identifying resources, assigning roles and responsibilities, and ensuring overall coordination. This process, especially the assessment

The authors are at the National Center for Infectious Diseases, Centers for Disease Control and Prevention, Atlanta, Georgia 30333, US. e-mail: Irotz@cdc.gov of needs and capabilities, is inherently problematic because it depends on theoretical probabilities, different scale considerations and impacts that are difficult to define. Unlike response planning for natural disasters, bioterrorism response planning lacks the advantage of referencing historical event-specific information. Therefore, planning efforts have often relied on 'what if' scenarios to project the impact of an event on a community.

As part of these efforts, multiple diseaseoutbreak models have been developed to estimate impact, use of resources and effectiveness of interventions ${ }^{1-7}$. In general, these models integrate clinical and epidemiological features of disease evident from previous outbreaks, intervention requirements or performance based on actual experiences or simulated terrorist attacks, and other parameters with unknown and assumed values.

Estimating the scope of a disease outbreak must include a consideration of factors such as population susceptibility, infective dose, incubation period, modes of transmission, duration of illness, mortality rate, effectiveness of treatment interventions and population movement. Contagious disease outbreaks have the added complexity of transmission parameters such as infectious periods and secondary attack rates. Disease-outbreak models must also quantify characteristics that have a range of potential values or have values that are determined on the basis of best-guess estimates or derived indirectly from other known parameters. No single model can take into account all factors, and most models strive to identify and incorporate the main factors influencing the specific outcome they are trying to predict, such as the number of secondary cases or the effectiveness of an intervention program ${ }^{5,6}$.

Modeling has also been used to quantify parameters that are used to develop guidance documents for community preparedness ${ }^{8}$. In addition, several computerized tools incorporating modeling and real-world experiences have been designed to assist public health and emergency-response planners in estimating resource needs during a bioterrorism event or an outbreak of a naturally occurring infectious disease (Table 1). But users of planning and response programs must understand the model's assumptions, parameters and predictive power so that the results are appropriately interpreted and applied. Moreover, although these tools can assist planners with predicting impact and resource needs in a naturally occurring outbreak or bioterrorism event, practical experience with simulated or realtime exercises and testing of response systems is essential and can help identify gaps that cannot be predicted through the use of these tools alone $\mathrm{e}^{9,10}$.

The Models of Infectious Disease Agent Study (MIDAS) project is a recent initiative supported by the US National Institutes of Health (NIH) to encourage the development of computational tools that can be used to model exposure to infectious pathogens, perform timely and cost-effective surveillance, model effectiveness and consequences of different interventions, and model decontamination of exposed facilities. The project involves multidisciplinary research and infor- 


\section{COMMENTARY}

Table 1 Examples of computerized tools for estimating outbreak-related resource needs

\begin{tabular}{lll}
\hline Tool & Description & Website \\
\hline Maxi-Vac & $\begin{array}{l}\text { Program for use in determining optimal staffing requirements for large-scale } \\
\text { smallpox vaccination clinics }\end{array}$ & $\begin{array}{l}\text { http://www.bt.cdc.gov/agent/smallpox/vaccination/ } \\
\text { maxi-vac/index.asp }\end{array}$ \\
\hline $\begin{array}{l}\text { Bioterrorism and Epidemic } \\
\text { Outbreak Response Model } \\
\text { (BERM) }\end{array}$ & $\begin{array}{l}\text { Program for estimating healthcare staffing needs for response to a bioterrorism } \\
\text { attack or large-scale natural disease outbreak, based on number of current staff } \\
\text { and number of patients expected to require rapid treatment }\end{array}$ \\
\hline FluSurge 1.0 & $\begin{array}{l}\text { Model for use in estimating hospital resource requirements during an influenza } \\
\text { pandemic and comparing existing hospital capacity with the numbers of persons } \\
\text { expected to require hospitalization, intensive care or ventilator support }\end{array}$ \\
\hline FluAid 2.0 & $\begin{array}{l}\text { Test version of software that provides a range of impact estimates for deaths, } \\
\text { hospitalizations and outpatient visits resulting from pandemic influenza }\end{array}$ \\
\hline
\end{tabular}

matics groups, and emphasizes informationdriven research rather than hypothesis-driven investigations for the development of these tools. After development and validation, these tools will reside in a centralized, publicly accessible database for use by policymakers, public health professionals and researchers (see URL at end of article). A steering committee of investigators with broad expertise in modeling, infectious diseases and public health will establish policies, set standards for data management, evaluate progress and provide a forum for the exchange of ideas within and beyond the MIDAS network. Leveraging and coordinating expertise and experience across multiple disciplines to set standards for the development and functionality of computerized modeling could greatly increase the usefulness of these tools. Evaluating these tools during outbreak planning and response activities not related to bioterrorism, as well as during simulated bioterrorism events, would further assist in determining their overall predictive capabilities.

\section{Detection}

Advances in detecting bioterrorism incidents involve concepts and systems designed to identify potential signals of an attack at the earliest possible stages. These systems target the early identification of moderate- to largescale outbreaks and may miss smaller events. These systems are meant to augment, not replace, reporting of diseases of interest or unusual or suspicious illnesses by healthcare providers and laboratory workers - the traditional first alert and line of defense against the spread of an infectious disease.

From a public health standpoint, detection systems can be separated into two general categories: systems that detect illness (syndromic, health-care utilization or diagnosis-based surveillance) or potential early indicators of illness (such as school absenteeism and drug sales), and systems that detect the release of a biological agent before the onset of symptoms in persons exposed (envi- ronmental monitoring) ${ }^{11}$. The main characteristics of these types of systems, along with their strengths and weaknesses, are described below.

Surveillance systems for early indicators of illness. Strategies used for early detection of illness have been designed around the increasing availability of electronic data. Examples of such data include emergency or nurse hotline calls, ambulance dispatch information, emergency department chief complaints, number of emergency department visits or hospital admissions, International Classification of Diseases, Ninth Revision (ICD-9) codes, over-the-counter medication sales and school absenteeism information. These data are used to identify illnesses or potential indicators of illness in the prediagnosis stage. Although ICD-9 codes can identify illness at the diagnosis stage, they are also being used in several surveillance systems to identify syndromes of illness that may be consistent with diseases caused by bioterrorism agents ${ }^{12-14}$.

Syndromic surveillance systems have been initiated by health departments in about 100 sites throughout the United States ${ }^{11}$, and similar systems have been implemented in other countries $^{15,16}$. A recent review found published information on 29 systems designed specifically for detecting bioterrorism-related diseases or syndromes ${ }^{17}$. Nine of these systems were syndromically based (Table 2), but most had not undergone full evaluations using public health guidelines for surveillance systems $^{18,19}$.

Other benefits for bioterrorism-related syndromic surveillance systems, such as natural outbreak detection, have been suggested and should also be considered when evaluating the utility of these systems ${ }^{20}$. Descriptions and formal evaluations of syndromic surveillance systems, as presented in the recently released reports from a 2003 US National Syndromic Surveillance Conference ${ }^{21}$, are essential to validate these methods for early event detection and to develop standards for future systems.

A moderate- or large-scale bioterrorism attack has not occurred in an area that has established a surveillance system specifically for its detection; therefore, most assessments of such systems have relied on their ability to detect nonbioterrorism outbreaks within the community ${ }^{22}$ or their performance using synthesized data ${ }^{13}$. In addition, only a few studies have been conducted examining the validity of the medical data collected for syndromic surveillance. Findings from two studies comparing syndromic categorization for respiratory-tract infections in the emergency department both for an established, hospitalbased surveillance system and for a shortterm, event-based system suggested that the use of emergency department discharge diagnoses with or instead of emergency department chief complaints may help increase the reliability of syndromic systems ${ }^{23,24}$. This finding is not surprising; discharge diagnoses tend to be assigned on the basis of additional diagnostic data, whereas chief complaints are assigned on the basis of the interpretation of presenting symptoms. For example, chest pain may be the chief complaint of a person whose condition is ultimately diagnosed as pneumonia after a chest radiograph. Such illnesses may not be categorized as a respiratory syndrome on the basis of the patient's chief complaint if chest pain was not part of the definition for respiratory syndromes. Standardization of syndromic case definitions in addition to the use of data from multiple sources could strengthen the usefulness and specificity of these systems.

Identifying unusual data trends or clusters is the goal of early event detection systems. Because many of the data streams used in these systems are nontraditional, historical data used to establish expected baselines may not be available. This has prompted the use of statistical methods capable of establishing baselines for aberration detection with limited previous data. Industry-proven qual- 


\section{COMMENTARY}

ity-control statistical methods to detect aberrations for cost savings have been adapted for use in several automated analysis tools developed for early event detection ${ }^{25,26}$. Alarms for aberration detection within analysis algorithms can be adjusted to increase or decrease the sensitivity of the system. Although the primary goal is early event detection, sensitivity must be adjusted and sound epidemiologic methods must be used to prevent false alarms, that can unnecessarily strain investigative resources and decrease confidence in the system.

After the attacks on the World Trade Center in September 2001, the New York City Department of Health and Mental Hygiene and the Centers for Disease Control and Prevention (CDC) initiated a four-week, active, emergency department-based syndromic surveillance system for disease outbreaks and illnesses compatible with diseases of concern for bioterrorism ${ }^{27}$. This system required substantial personnel resources for data collection; however, it allowed the Department of Health and Mental Hygiene to establish an active mechanism for bioterrorism event monitoring when routine surveillance and communication systems were disrupted. Data were analyzed on the basis of temporal or spatial syndrome clustering, resulting in eight citywide temporal and 24 spatial cluster alarms that required addi- tional evaluation. No outbreaks were identified in the follow-up investigations of these alarms.

None of the four cutaneous anthrax cases associated with the intentional distribution of contaminated mail that subsequently occurred in New York during the surveillance period were identified through this system; however, only one of the affected individuals visited an emergency department before diagnosis. After the single case of inhalational anthrax was diagnosed, the system did not show increases in emergency department visits for respiratory illness-information that allowed officials to reassure the public during the follow-up investigation.

This system has since been adapted to function as an automated, electronic emergency department syndromic surveillance system to improve its acceptance and sustainability for long-term use ${ }^{28}$. Similar systems have also been deployed to support short-term, enhanced bioterrorism surveillance needs for special events that are considered potential targets for terrorism ${ }^{24,29,30}$. Additional systems that further automate data collection and analysis have been developed to improve acceptance and sustainability (Box 1).

Several efforts to improve international surveillance have focused on strengthening reporting mechanisms by creating electronically linked networks to enhance information sharing and communications globally among healthcare providers, public health authorities and, in some cases, nontraditional partners. The Global Outbreak Alert and Response Network formalized in 2000 by the World Health Organization (WHO) compiles outbreak information from more than 250 WHO collaborating centers, United Nations agencies, ministries of health, laboratories, universities and other surveillance systems such as Health Canada's Global Public Health Intelligence Network and the US Department of Defense Global Emerging Infectious System and shares this information with partners throughout more than 110 networks internationally ${ }^{31}$. This expansive network identified and verified 578 disease outbreaks in 132 countries from July 1998 to August 2001, and had an important role in international surveillance, communication and response during the 2003 global outbreak of severe acute respiratory syndrome (SARS) ${ }^{31,32}$.

Environmental monitoring systems. Detecting the release of a biological agent before the onset of symptoms in exposed subjects is the goal of environmental monitoring systems. Such systems have been developed for the detection of agent release in indoor and outdoor settings. These systems use testing methods for rapid agent identification based on real-time polymerase chain reaction (PCR) or

Table 2 Comparison of bioterrorism surveillance systems ${ }^{a}$

\begin{tabular}{|c|c|c|c|c|}
\hline System Name & Syndromes & Originator & Data collection & Evaluation \\
\hline $\begin{array}{l}\text { Border Infectious Disease } \\
\text { Surveillance Project (BIDS) }\end{array}$ & Hepatitis; vesicular rash; influenza/respiratory illness & $\mathrm{CDC}$ & $\begin{array}{l}\text { Additional data } \\
\text { collection required }\end{array}$ & None \\
\hline $\begin{array}{l}\text { Early Warning Outbreak } \\
\text { Recognition System }\end{array}$ & $\begin{array}{l}\text { Fever; watery diarrhea; bloody diarrhea; bleeding; } \\
\text { difficulty breathing; jaundice; vomiting; cough; paralysis; } \\
\text { unconsciousness; intradermal hemorrhage }\end{array}$ & $\begin{array}{l}\text { Indonesian Ministry } \\
\text { of Health }\end{array}$ & $\begin{array}{l}\text { Additional data } \\
\text { collection required }\end{array}$ & None found \\
\hline ESSENCE & $\begin{array}{l}\text { Respiratory illness, gastrointestinal illness; fever; } \\
\text { neurologic syndromes; dermatologic-infectious syndromes; } \\
\text { dermatologic hemorrhagic syndromes; coma; sudden death }\end{array}$ & US Department of Defense & $\begin{array}{l}\text { Automated capture of } \\
\text { ICD-9 codes }\end{array}$ & In process \\
\hline $\begin{array}{l}\text { Health Buddy and the } \\
\text { Biothreat Active Surveillance } \\
\text { Integrated Information and } \\
\text { Communication System }\end{array}$ & Customizable to track syndromes of interest & Health Hero Network, Inc. & $\begin{array}{l}\text { Additional data } \\
\text { collection required }\end{array}$ & None found \\
\hline LEADERS & Customizable to track syndromes of interest & Idaho Technology, Inc. & $\begin{array}{l}\text { Additional data } \\
\text { collection required }\end{array}$ & None found \\
\hline $\begin{array}{l}\text { National Health Service } \\
\text { Direct }\end{array}$ & Various syndromes of interest & United Kingdom & $\begin{array}{l}\text { Additional data } \\
\text { collection required }\end{array}$ & $\begin{array}{l}\text { Peer-reviewed } \\
\text { reports }\end{array}$ \\
\hline $\begin{array}{l}\text { Rapid Syndrome } \\
\text { Validation Project }\end{array}$ & $\begin{array}{l}\text { Flu-like illness; fever with skin findings; fever with altered } \\
\text { mental status; acute bloody diarrhea; acute hepatitis; } \\
\text { acute respiratory distress syndrome }\end{array}$ & $\begin{array}{l}\text { Sandia National } \\
\text { Laboratories }\end{array}$ & $\begin{array}{l}\text { Additional data } \\
\text { collection required }\end{array}$ & In process \\
\hline $\begin{array}{l}\text { Syndromal Surveillance } \\
\text { Tally Sheet }\end{array}$ & $\begin{array}{l}\text { Flu-like symptoms; fever with mental status changes; } \\
\text { fever with skin rash; diarrhea with dehydration; visual or } \\
\text { swallowing difficulties; drooping eyelids; slurred speech or } \\
\text { dry mouth; acute respiratory distress syndromes }\end{array}$ & $\begin{array}{l}\text { Santa Clara County Public } \\
\text { Health Department, } \\
\text { California, US }\end{array}$ & $\begin{array}{l}\text { Additional data } \\
\text { collection required }\end{array}$ & In process \\
\hline $\begin{array}{l}\text { Syndromic Surveillance } \\
\text { Using Automated Records }\end{array}$ & ICD-9 codes & Boston & $\begin{array}{l}\text { Automated capture of } \\
\text { ICD-9 codes }\end{array}$ & $\begin{array}{l}\text { Peer-reviewed } \\
\text { report }\end{array}$ \\
\hline
\end{tabular}

anformation adapted from ref. 17. 


\section{BOX 1 AUTOMATED SURVEILLANCE SYSTEMS}

ESSENCE II (Electronic Surveillance System for the Early Notification of CommunityBased Epidemics) is a system that strives to fully automate data collection, processing, analysis and reporting functions, while using multiple data sources ${ }^{13}$. Clinical data (such as ICD-9 codes, hospital emergency department chief complaints, veterinary reports) are grouped into syndromes and analyzed along with preclinical data (prescription medications, school absenteeism). This system is an evolving prototype, and is being evaluated and refined while being used for surveillance activities.

BioSense, a recent initiative from the Department of Health and Human Services and the Department of Homeland Security, enables electronic transmission of pre-existing diagnostic and prediagnostic health data to CDC from national health data sources (http://www.cdc.gov/phin/Webinars/BioSense.htm). One of the most important goals of this initiative is to promote standards for national data vocabulary and messaging along with other specifications to allow for integration with other public health systems. Ensuring unifying frameworks for newly developed health and environmental surveillance information systems will enable health authorities to integrate information from multiple systems, enhancing detection and decision-making capabilities. Although BioSense is designed to establish electronic links between the CDC and national health data sources, its primary purpose is to provide information obtained from these national sources back to state and local public health jurisdictions as a complement to their existing local public health surveillance activities.

Systems such as RODS (Real-time Outbreak and Disease Surveillance system) provide state and local public health authorities similar electronic linkages and analysis of available clinical data from local clinical and other sources ${ }^{30}$. Although developed for the early detection of potential bioterrorist threats, both systems are designed to be equally useful in detecting early signals of naturally occurring disease outbreaks.

antibody-assay methodologies. Programs such as BioWatch and BASIS (Biological Aerosol Sentry and Information System) use sampling stations to periodically pull air samples through a filter, which is then analyzed. These systems perform with minimal false alarms, but require personnel resources to collect and test the filters ${ }^{33,34}$. Automation of sample preparation and testing can address some of the human resource issues presented by these systems, as has been shown by a recently developed, semiautomated version of a BASIS laboratory, which allows two technicians to complete 10,000 PCR assays on 1,000 samples in an eight-hour period ${ }^{34}$.

The ultimate goal of environmental testing is a fully automated and sensitive system with low maintenance requirements and few false alarms. The Biohazard Detection System (BDS), currently being used by the US Postal Service, tests the sample within the air collection apparatus, avoiding the personnel requirements for routine collection, transportation and laboratory testing of the sample $^{35}$. But positive alarms from this system must still undergo confirmation by traditional laboratory methods. This requirement is likely to remain in effect until more experience is gained regarding the system's performance in the field. Similarly, the Autonomous Pathogen Detection System (APDS) is a fully automated system that can perform 100 simultaneous measurements every 30 minutes for more than a week and has the capacity to detect about 30 different pathogens ${ }^{34,36}$.

The reliability of environmental systems in accurately detecting a biological agent released into the environment is affected by many factors, including the specificity of the testing methods, the efficiency of the collection apparatus, weather patterns, location and placement of the monitors, and the concentration of the organism in the air sampled by the collector. These and other technical factors must be considered when deploying an environmental monitoring system. Monitoring systems using PCR and antibodybased systems have greater sensitivity and specificity than other methods for biological agent detection ${ }^{34}$. But these systems cannot assess the viability of the organism, and their sensitivity must balance the need for detection with the resource demands and system distrust that would result from frequent false alarms. So far, the BioWatch system has tested thousands of environmental air samples with no false positives (J. M. Miller, personal communication). But these systems also detect targeted organisms naturally present in the environment, as was determined to have occurred in the Houston area after several positive signals for Francisella tularensis were obtained $^{37,38}$.
Training, communication and response protocols, along with standards for data interpretation and reporting, should be established and coordinated with response authorities in conjunction with the deployment of these systems to fully optimize their role as a potential early-detection tool.

\section{Diagnosis}

Rapid and accurate diagnosis of potential bioterrorism agents is crucial for identifying and lessening the impact of an infectious threat. A major effort to enhance diagnostic capacity is the Laboratory Response Network (LRN), established by the CDC in collaboration with the Association of Public Health Laboratories, the Federal Bureau of Investigation and other partners to respond quickly to acts of chemical and biological terrorism, emerging infectious diseases and other public health threats and emergen$\operatorname{cies}^{39}$. This diagnostic network became operational in 1999 and now links more than 120 federal, state and local reference laboratories, as well as private and international laboratories working across the human, veterinary, agricultural and environmental fields. The varying specialties and levels of capacity of these laboratories enable them to rapidly recognize, rule out, confirm and characterize agents of bioterrorism and other infectious agents (Fig. 1).

Molecular and antibody-based diagnostics form the basis for tests developed for rapidly identifying potential bioterrorism agents in clinical and environmental samples. Realtime PCR and time-resolved fluorescence immunoassays are two techniques used by the LRN to detect several CDC Category A and $\mathrm{B}$ agents, that is, biological agents given highest priority on the basis of their potential public health impact ${ }^{40}$. These techniques have decreased the time for identification of an agent from days (using traditional, cul-

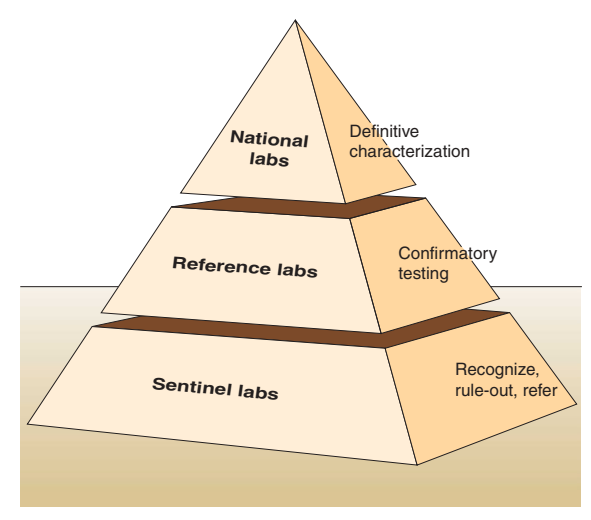

Figure 1 Operational components of the Laboratory Response Network. 


\section{COMMENTARY}

ture-based methods) to hours. Real-time PCR allows both the amplification and detection of genetic material in a single procedure, and use of multiple genetic probes for a particular agent further increases the specificity of the test. Time-resolved fluorescence immunoassays offer an advantage over immunoradiometric assays by using nonradioisotopic procedures.

During the public health response to the anthrax letters in 2001, the LRN tested more than 125,000 clinical and environmental samples using the real-time PCR assay developed for Bacillus anthracis. The test performed with $100 \%$ specificity on cultures and clinical samples, and was invaluable in rapidly confirming or excluding the diagnosis of anthrax in patients with compatible symptoms ${ }^{41}$. More recently, after the global outbreak of SARS, LRN reference laboratories developed new diagnostic PCR assays and rapidly distributed validated reagents and protocols to LRN laboratories within weeks of the discovery of the causative agent. This technology is also used by the LRN laboratories analyzing filters from environmental monitors placed in densely populated areas as part of the BioWatch program.

Multiplexed assays allow simultaneous testing for multiple biological agents from a single sample. Multiplex gene expression analysis has been used in genomics to validate existing targets, identify new targets and establish genetic causes of disease ${ }^{42,43}$. Liquid array-based multiplexed immunoassays use microbeads for simultaneous detection of multiple biological agents and can perform in high-throughput mode with a sensitivity comparable to that of the standard enzymelinked immunosorbent assay ${ }^{36,44,45}$. Future LRN use of this technology will further advance the rapid screening capabilities for potential bioterrorism agents in both clinical and environmental specimens.

\section{Response}

Earlier detection of a bioterrorism attack provides an opportunity for rapid intervention to mitigate its impact on a community. Interventions include the identification of affected subjects, investigations to determine the source of the outbreak and people at risk, and the implementation of control measures to contain and stop further spread. Tools that can assist with the rapid initiation of these response activities are essential for public health and medical authorities.

Identification of affected subjects. Rapidly identifying and initiating treatment of potentially affected individuals after the intentional release of a biological agent is of primary importance in a public health response to bioterrorism. Obtaining accurate information on areas potentially exposed during a release and the estimated agent concentrations within the area is important for prioritizing response resources and therapeutic interventions. As an example, integration of Geographic Information System mapping capabilities into the environmental investigation of the Trenton, New Jersey, postal facility after the 2001 anthrax letters provided spatial representation of contamination and valuable information on the areas of potential exposure $^{46}$. Integrating weather pattern information and plume modeling could enhance this information to support environmental monitoring. The National Atmospheric Release Advisory Center has developed integrated meteorological and dispersion model systems that can assist local emergency response authorities in developing forecast plume models after an identified release. Continued evaluation of these systems with exercises and outbreak investigations, however, is still necessary to enhance familiarity with these tools and foster confidence in their ability to provide useful information to guide decisions during a response.

Outbreak investigation. During the investigation of the US anthrax letters in 2001, a centralized repository was developed for epidemiological, informational and educational materials to assist with the multiple simultaneous field investigations ${ }^{47}$. This repository enabled greater coordination and efficiency in subsequent investigations. Development and testing of similar, ready-to-use toolkits for other potential bioterrorism agents with standardized database templates for information collection would facilitate rapid public health investigations of outbreaks involving these organisms.

The Florida Department of Health has developed a web-based system for disease reporting, surveillance and analysis activities called MERLIN. MERLIN links multiple local health department investigative activities during an outbreak and assists with data collection and analysis, information sharing and overall coordination of a response. A formal evaluation and more in-depth description of this system's capabilities for these activities would be beneficial. In the absence of performance evaluation during an actual bioterrorism response, existing systems such as MERLIN must be assessed through naturally occurring outbreaks and bioterrorism exercises.

Many potential bioterrorism agents also cause endemic disease, making early identifi- cation of an intentional outbreak difficult ${ }^{48}$. Multiple criteria have been suggested for use during an investigation that may help differentiate between intentional and unintentional outbreaks ${ }^{49-52}$. Grunow and Finke have combined various criteria and developed a semiquantitative method for assessing the likelihood that an outbreak is intentional $(\text { Table } 3)^{53}$. This method assesses multiple nonconclusive and/or conclusive criteria that take into consideration epidemiological and clinical characteristics, specific pathogen features, political, military and social characteristics of the affected area, and any direct evidence of a biological weapon or proof of release. Data from field epidemiological, laboratory and clinical sources are required to apply this assessment methodology. So, it would be used during the investigation of an outbreak, or retrospectively to determine the likelihood of a natural versus intentional source. It will be necessary to apply this technique to assess other outbreaks in order to further evaluate its ability to distinguish between likely and unlikely bioterrorism events. If validated, this method could be useful in assessing suspicious outbreaks, particularly if the process were automated to allow rapid scoring as information is collected during an investigation.

Large-scale responses have extensive informatics needs, as was evident during the 2001 anthrax letters event and the 2003 SARS outbreak. Several authors have further outlined these needs on the basis of public health and medical decision-making requirements in simulated bioterrorism exercises and in actual bioterrorism and nonbioterrorism outbreak responses ${ }^{46,54}$. A review found 217 information technology decision-support systems that may be of potential use to clinicians and public health officials during a bioterrorism $\operatorname{attack}^{29}$. These systems included detection and diagnostics, management and prevention, surveillance, reporting and communication, and integrated surveillance, communication, command and control. Most were not specifically designed for bioterrorism, and the few that were principally addressed detection and integrated command-and-control activities.

Control measures. Efforts to improve intervention capabilities to prevent or treat disease after the release of a biological agent have included the development of national stockpiles of medications, vaccines and medical supplies, programs to assist with rapid community distribution, and legislation to assist with the development of and access to new therapeutic countermeasures. The Strategic National Stockpile assures accessibility to 


\section{COMMENTARY}

Table 3 Criteria used for differentiating natural and intentional outbreaks involving biological warfare agents ${ }^{\mathrm{a}}$

Criteria

Nonconclusive

Existence of biological risk

Existence of biological threat

Special aspects of agent

Peculiar geographic distribution

High environmental concentrations of agent

Peculiar intensity or dynamic of outbreak

Peculiarities in transmission mode

Peculiarities of timing

Unusually rapid spread

Limitation of outbreak to specific population

Peculiarities of clinical manifestation

Conclusive

Identification as a biological warfare agent

Proof of release by biological weapon
Description of criteria

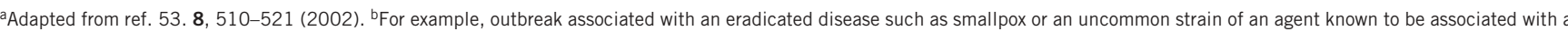
biological weapons program

large amounts of known medical countermeasures for high-priority bioterrorist agents, and the coupling of this stockpile with rapid transportation mechanisms provides ready delivery capability to areas of need. Distribution of countermeasures once they have arrived in a community, however, is still a difficult task for local authorities. The Cities Readiness Initiative, a pilot program in several cities that integrates multiple delivery mechanisms to enable rapid and broad delivery of countermeasures, has been recently initiated and, if successful, will provide community-level examples of robust and well-functioning delivery plans and capabilities to help guide planning efforts.

Several potential high-priority bioterrorism agents have limited or no known therapeutic or chemoprophylactic options. Since late 2001, about $\$ 5$ billion has been allotted by the US government for research and development of medical countermeasures ${ }^{55}$. Other efforts to address this need for new or improved drugs, vaccines and diagnostics include recent Congressional approval of the BioShield Act, which promotes the development and emergency use of these countermeasures. Although this is an important step, additional actions are needed to enhance research and development of new therapeutics. Examples include coordinating national biodefense countermeasure development, increasing incentives to engage private industry, and exploration and potential regulatory acceptance of new methods for candidate drug evaluation (such as animal safety and efficacy models, and computer modeling for toxicity and pharmacokinetics) ${ }^{56}$.

\section{Summary}

Improving our ability to detect and respond to a bioterrorist threat continues to be a major public health priority. New programs and systems to improve detection of bioterrorist events should be designed to augment and complement existing strategies and safeguards. In addition, specific research agendas that include efforts to improve diagnostics, therapies and vaccines for potential bioterrorism agents are particularly needed. As new strategies for addressing these threats are developed and refined, much can be learned from examining efforts to track and stop a naturally occurring infectious disease outbreak. Of primary importance is the need to be alert for unusual signs or symptoms that can represent the initial clues of an outbreak. Such vigilance for the unexpected has historically proven invaluable. Recognition of unusually severe respiratory disease by observant clinicians signaled outbreaks of hantavirus pulmonary syndrome in 1993 and SARS in 2003; an increase in orders to treat an unusual form of pneumonia noted by an alert technician hinted at the first cases of AIDS in 1981; suspicion of anthrax by astute clinical and laboratory staff in Florida suggested a possible bioterrorism event in 2001 .

Many other important lessons learned from recent infectious disease outbreaks also have direct relevance for bioterrorism preparedness activities. The need to increase national and global partnerships is particularly important. Such collaborative activities will strengthen preparedness and response efforts by expanding the 'radar screen' and establishing essential connections across multiple specialties to enable real-time detection and communication of critical information. Finally, as with naturally occurring infectious diseases, efforts are needed to understand and address the root causes of these threats and to enhance the political will and personal actions needed to lessen the likelihood of their occurrence and mitigate their impact.

URLs. BioShield Act http://www.whitehouse.gov/bioshield/

Cities Readiness Initiative

http://www.bt.cdc.gov/cri/

Health Canada's Global Public Health Intelligence Network http://gphin-rmisp. hc-sc.gc.ca/index.html

MERLIN http://www.doh.state.fl.us/ disease\%5Fctrl/epi/topics/merlininfo.htm

Models of Infectious Disease Agent Study (MIDAS) http://www.nigms.nih.gov/research/ midas.html

US Department of Defense Global Emerging Infectious System http://www.geis.fhp.osd.mil/ US National Atmospheric Release Advisory Center http://narac.llnl.gov/

Strategic National Stockpile http://www.bt.cdc.gov/stockpile/index.asp.

\section{ACKNOWLEDGMENTS}

The authors thank A. Fleischauer, B. Garza, M. Meltzer, R. Meyer, R. Moseley, D. Nichelson, M. Taylor, A. Greenspan, M. Miller and T. Treadwell for their assistance in preparing the manuscript.

\section{COMPETING INTERESTS STATEMENT}

The authors declare that they have no competing financial interests.

1. Meltzer, M.L., Damon, L., LeDuc, J.W. \& Millar, J.D. Modeling potential responses to smallpox as a bioterrorist weapon. Emerg. Infect. Dis. 7, 959-969 (2001).

2. Klein, R.W., Dittus, R.S., Roberts, S.D. \& Wilson, J.R. Simulation modeling and health-care decision mak- 


\section{COMMENTARY}

ing. Med. Decis. Making 13, 347-354 (1993).

3. Kaufmann, A.F., Meltzer, M.I. \& Schmid, G.P. The economic impact of a bioterrorist attack: are prevention and postattack intervention programs justifiable? Emerg. Infect. Dis. 3, 83-94 (1997).

4. Walden, J. \& Kaplan, E.H. Estimating time and size of bioterror attack. Emerg. Infect. Dis. 10, 1202-1205 (2004).

5. Brookmeyer, R., Johnson, E. \& Bollinger, R. Modeling the optimum duration of antibiotic prophylaxis in an anthrax outbreak. Proc. Natl. Acad. Sci. USA 100 10129-10132 (2003).

6. Gani, R. \& Leach, S. Epidemiologic determinants for modeling pneumonic plague outbreaks. Emerg. Infect. Dis. 10, 608-614 (2004).

7. Wein, L.M., Craft, D.L. \& Kaplan, E.H. Emergency response to an anthrax attack. Proc. Natl. Acad. Sci. USA 100, 4346-4351 (2003).

8. Community-based mass prophylaxis: a planning guide for public health preparedness (Agency for Healthcare Research and Quality, Rockville, Maryland, USA 2004). http://www.ahrq.gov/research/cbmprophyl/cbmpro.htm

9. Andress, K. A postevent smallpox mass vaccination clinic exercise. Disaster Manag. Response 1, 54-58 (2003).

10. O'Toole, T., Mair, M. \& Inglesby, T.V. Shining light on "Dark Winter". Clin. Infect. Dis. 34, 972-983 (2002).

11. Buehler, J.W., Berkelman, R.L., Hartley, D.M. \& Peters, C.J. Syndromic surveillance and bioterrorismrelated epidemics. Emerg. Infect. Dis. 9, 1197-1204 (2003).

12. Pavlin, J.A. \& Kelley, P.W. ESSENCE: Electronic Surveillance System for the Early Notification of Community-based Epidemics (U. S. Department of Defense, Global Emerging Infections Surveillance and Response System, Silver Spring, Maryland, USA, 2001).

13. Lombardo, J. et al. A systems overview of the Electronic Surveillance System for the Early Notification of Community-Based Epidemics (ESSENCE II). J. Urban Health 80, i32-i42 (2003).

14. Lober, W.B. et al. Syndromic surveillance using automated collection of computerized discharge diagnoses. J. Urban Health 80, i97-i106 (2003).

15. Takahashi, H., Fujii, H., Shindo, N. \& Taniguchi, K. Evaluation of the Japanese school health surveillance system for influenza. Jpn. J. Infect. Dis. 54, 27-30 (2001).

16. Quenel, P. \& Dab, W. Influenza A and B epidemic criteria based on time-series analysis of health services surveillance data. Eur. J. Epidemiol. 14, 275-285 (1998).

17. Bravata, D.M. et al. Systematic review: surveillance systems for early detection of bioterrorism-related diseases. Ann. Intern. Med. 140, 910-922 (2004).

18. Centers for Disease Control and Prevention. Updated guidelines for evaluating public health surveillance systems. Recommendations from the Guidelines Working Group. MMWR Recomm. Rep. 50, 1-35 (2001).

19. Centers for Disease Control and Prevention. Framework for evaluating public health surveillance systems for early detection of outbreaks: recommendations from the CDC Working Group. MMWR Recomm. Rep. 53, 1-11 (2004).

20. Reingold, A. If syndromic surveillance is the answer, what is the question? Biosecur. Bioterror. 1, 77-81 (2003).

21. Centers for Disease Control and Prevention. Syndromic Surveillance: Reports from a National Conference, 2003. MMWR Morb. Mortal. Wkly. Rep. 53 (2004).

22. Mostashari, F., Fine, A., Das, D., Adams, J. \& Layton, M. Use of ambulance dispatch data as an early warning system for communitywide influenzalike illness, New York City. J. Urban Health 80, i43-i49 (2003).

23. Reis, B.Y. \& MandI, K.D. Syndromic surveillance: the effects of syndrome grouping on model accuracy and outbreak detection. Ann. Emerg. Med. 44, 235-241 (2004).

24. Fleischauer, A.T. \& Silk, B.J. The validity of chief complaint and discharge diagnosis in emergency department-based syndromic surveillance. Acad. Emergency Med. (in the press).

25. Centers for Disease Control and Prevention. The state of statistical process control as we proceed into the 21st century. J. Am. Statist. Assoc. 95, 992-998 (2000).

26. Hutwagner, L., Thompson, W., Seeman, G.M. \& Treadwell, T. The bioterrorism preparedness and response Early Aberration Reporting System (EARS). J. Urban Health 80, i89-i96 (2003).

27. Das, D. et al. Enhanced drop-in syndromic surveillance in New York City following September 11 2001. J. Urban Health 80, i76-i88 (2003).

28. Heffernan, R. et al. Syndromic surveillance in public health practice, New York City. Emerg. Infect. Dis. 10 858-864 (2004).

29. Bravata, D.M. et al. Bioterrorism preparedness and response: use of information technologies and decision support systems. Evid. Rep. Technol. Assess. 1-8 (2002).

30. Tsui, F.C. et al. Technical description of RODS: a realtime public health surveillance system. J. Am. Med. Inform. Assoc. 10, 399-408 (2003).

31. Heymann, D.L. \& Rodier, G.R. Hot spots in a wired world: WHO surveillance of emerging and re-emerging infectious diseases. Lancet Infect. Dis. 1, 345-353 (2001).

32. Heymann, D.L. \& Rodier, G. Global surveillance, national surveillance, and SARS. Emerg. Infect. Dis. 10, 173-175 (2004).

33. Fitch, J.P. et al. Rapid development of nucleic acid diagnostics. Proc. IEEE 90, 1780-1721 (2002).

34. Fitch, J.P., Raber, E. \& Imbro, D.R. Technology challenges in responding to biological or chemical attacks in the civilian sector. Science 302, 1350-1354 (2003).

35. Centers for Disease Control and Prevention. Responding to detection of aerosolized Bacillus anthracis by autonomous detection systems in the workplace. MMWR Recomm. Rep. 53, 1-12 (2004).

36. McBride, M.T. et al. Multiplexed liquid arrays for simultaneous detection of simulants of biological warfare agents. Anal. Chem. 75, 1924-1930 (2003).

37. Houston Department of Health and Human Services \& Harris County Public Health and Environmental Services. Officials following up on bacteria detection (press release) October 9 (2004). http://www.ci.houston.tx.us/departme/health/bacteria\%20detection.htm

38. Berger, E. Suspicious bacteria detected; Security monitors spot germ; terrorism discounted. The Houston (TX) Chronicle, 10 Oct (2003).

39. Morse, S.A. et al. Detecting biothreat agents: the
Laboratory Response Network. ASM News 69, 433-437 (2003).

40. Rotz, L.D., Khan, A.S., Lillibridge, S.R., Ostroff, S.M. \& Hughes, J.M. Public health assessment of potential biological terrorism agents. Emerg. Infect. Dis. 8, 225-230 (2002).

41. Hoffmaster, A.R. et al. Evaluation and validation of a real-time polymerase chain reaction assay for rapid identification of Bacillus anthracis. Emerg. Infect. Dis. 8, 1178-1182 (2002)

42. Golub, T.R. et al. Molecular classification of cancer: class discovery and class prediction by gene expression monitoring. Science 286, 531-537 (1999).

43. Gerhold, D.L., Jensen, R.V. \& Gullans, S.R. Better therapeutics through microarrays. Nat. Genet. 32, 547-551 (2002).

44. Langlois, R.G. et al. Development of an autonomous pathogen detection system in the Proceedings of the Joint Conference on Point Detection for Chemical and Biological Defense (Williamsburg, Virginia, 2000).

45. Rao, R.S. et al. Comparison of multiplexed techniques for detection of bacterial and viral proteins. J. Proteome. Res. 3, 736-742 (2004).

46. Zubieta, J.C., Skinner, R. \& Dean, A.G. Initiating informatics and GIS support for a field investigation of bioterrorism: the New Jersey anthrax experience. Int. J. Health Geogr. 2, 8 (2003).

47. Reissman, D.B., Steinberg, E.B., Magri, J.M. \& Jernigan, D.B. The anthrax epidemiologic tool kit: an instrument for public health preparedness. Biosecur. Bioterror. 1, 111-116 (2003).

48. Torok, T.J. et al. A large community outbreak of salmonellosis caused by intentional contamination of restaurant salad bars. JAMA 278, 389-395 (1997).

49. Steyn, B. in Scientific and Technical Means of Distinguishing Between Natural and Other Outbreaks of Disease (eds. Dando, M., Pearson, G. S. \& Kriz, B.) 87-92 (Kluwer Academic Publishers, Dordrecht, The Netherlands, 2001)

50. Noah, D.L., Sobel, A.L., Ostroff, S.M. \& Kildew, J.A. Biological warfare training: infectious disease outbreak differentiation criteria. Mil. Med. 163, 198-201 (1998).

51. Treadwell, T.A., Koo, D., Kuker, K. \& Khan, A.S. Epidemiologic clues to bioterrorism. Public Health Rep. 118, 92-98 (2003).

52. LeDuc, J.W., Ostroff, S.M., McDade, J.E., Lillibridge, S. \& Hughes, J.M. in Emerging Infections (eds. Scheld, W.M., Craig, W.A. \& Hughes, J.M.) 219-230 (ASM Press, Washington, DC, 1999).

53. Grunow, R. \& Finke, E.J. A procedure for differentiating between the intentional release of biological warfare agents and natural outbreaks of disease: its use in analyzing the tularemia outbreak in Kosovo in 1999 and 2000. Clin. Microbiol. Infect. 8, 510-521 (2002)

54. Bravata, D. M. et al. A conceptual framework for evaluating information technologies and decision support systems for bioterrorism preparedness and response. Med. Decis. Making 24, 192-206 (2004).

55. Schuler, A. Billions for biodefense: federal agency biodefense funding, FY2001-FY2005. Biosecur. Bioterror. 2, 86-96 (2004).

56. Gilfillan, L. et al. Taking the measure of countermeasures: leaders' views on the nation's capacity to develop biodefense countermeasures. Biosecur. Bioterror. 2, 1-8 (2004). 